EESTI NSV TEADUSTE AKADEEMIA TOIMETISED. 29. KOIDE FUOSIKA * MATEMAATIKA. 1980, NR. 3

ИЗВЕСТИЯ АКАДЕМИИ НАУК ЭСТОНСКОИ ССР. ТОМ 29 ФИЗИКА * МАТЕМАТИКА. 1980, №3 3

\title{
ГОРЯЧАЯ ЯДЕРНАЯ ФЛЮОРЕСЦЕНЦИЯ, ВОЗБУЖДАЕМАЯ СИНХРОТРОННЫМ ИЗЛУЧЕНИЕМ
}

V. HIZNJAKOV, M. HAAS. SONKROTRONKIIRGUSSEGA ERGASTATUD KUUM TUUMAFLUORESTSENTS

V. HIZHNYAKOV, M. HAAS. HOT NUCLEAR FLUORESCENCE EXCITED BY SYNCHROTRON RADIATION

Синхротронное излучение (СИ) ввиду его исключительно высокой яркости в коротковолновом диапазоне может служить эффективным средством возбуждения ядерных $\gamma$-уровней $\left[{ }^{1}\right]$. Это позволяет использовать СИ для решения различных задач ядерной спектроскопии $\left[{ }^{1,2}\right]$. Цель данной работы - рассмотреть возможности изучения с помощью СИ горячей ядерной флюоресценции (ГЯФ) в кристаллах. Нас будут интересовать $\gamma$-уровни с энергией $E_{0} \sim 0,1-1$ Мэв и временем жизни $\tau_{0} \sim 10^{-10}-10^{-14}$ сек.

ГЯФ есть часть спектра резонансного рассеяния $\gamma$-квантов, связанная с испусканием вторичных фотонов «горячим», т. е. быстро двигающимся ядром в условиях затухшей корреляции фаз возбуждающего кванта и ядра $\left[{ }^{3}\right]$. Избыточную (в сравнении с тепловой) кинетическую энергию ядро приобретает при поглощении $\gamma$-кванта в фононном крыле; эта энергия в среднем равна энергии отдачи $R=E_{0}^{2} / 2 M c^{2}$ (M - масса ядра, c - скорость света). Для резонансов с энергией $E_{0} \simeq 0,3$ Мэв и $A \simeq 50$ она равна $\sim 1$ эB $(A-$ массовое число $)$. Время затухания указанной выше корреляции фаз при возбуждении СИ определяется длительностью $\left(\tau_{\text {им }}\right)$ импульсов СИ, $\tau_{\text {им }} \sim 10^{-20}$ сек. Это время гораздо меньше как времени жизни возбужденного ядра $\tau_{0}$, так и времени релаксации его кинетической энергии $\tau_{э н} \sim$ $\sim 10^{-11}-10^{-12}$ сек. Поэтому основная часть резонансно рассеянных $\gamma$-квантов в рассматриваемом случае есть ГЯФ.

Характерная особенность спектра ГЯФ - большая (в сравнении c шириной обычной ядерной флюоресценции) ширина $\Gamma_{\text {гф }} \sim$ $\sim 3,5 R|\cos \theta|$, где $\theta-$ угол рассеяния (см. рисунок). При этом заметная часть указанного спектра уже при комнатной температуре может перекрываться со спектром поглощения. Это позволяет изучать ГЯФ с помощью резонансного поглощения, т. е. без использования спектрометра. Сказанное особенно важно, поскольку относительное разрешение спектрометра должно быть не хуже $E / M c^{2} \leqslant 10^{-5}-10^{-6}$, что в настоящее время, по-видимому, трудно достижимо.

Рассмотрим прежде всего резонансы с $E_{0} \sim 0,2-1$ Мэв и $\tau_{0} \sim$ $\sim 10^{-11}-10^{-14}$ сек. Одна из возможных схем эксперимента, позволяющего изучать ГЯФ таких $\gamma$-уровней, состоит в следующем. СИ направляется на кристаллическую мишень толщиной $0,1-1$ мм, содержащую резонансные ядра в качестве основного вещества, Рассеянные назад 


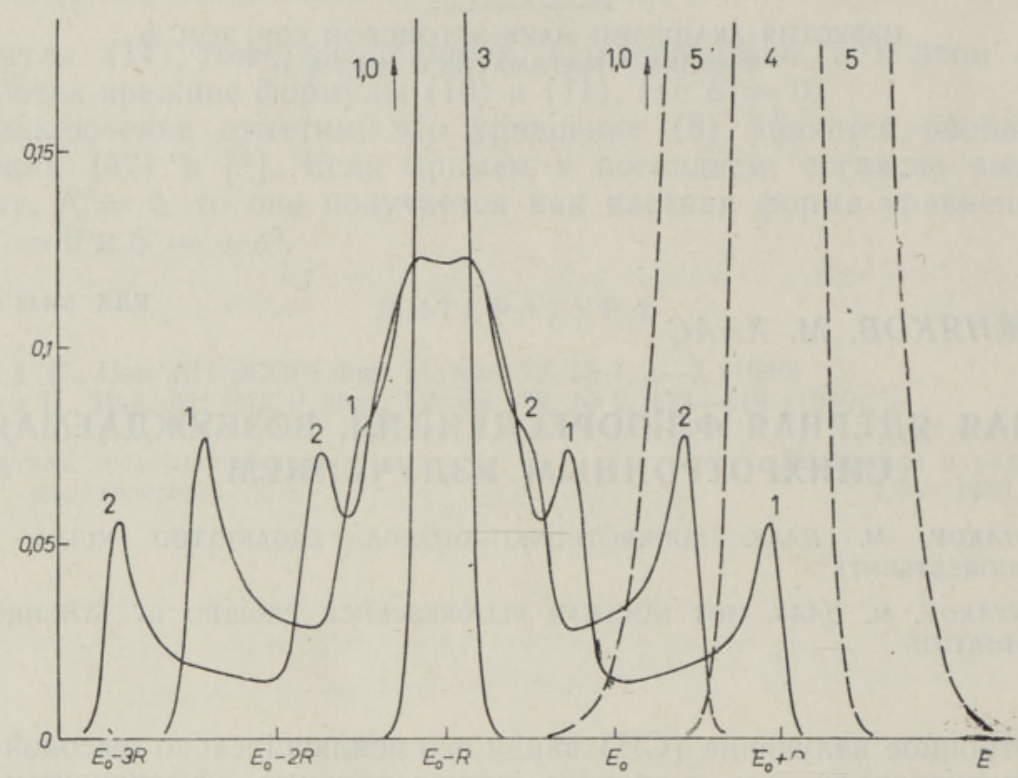

Спектры резонансного рассеяния и поглощения $\gamma$-квантов в кристалле в дебаевской модели колебаний ядер. $R=110 \hbar \omega_{D}, \omega_{D} \tau_{0}=10$, - спектр рассеяния, $T=0 \mathrm{~K}:$ 1) $\theta=0^{\circ}$, 2) $\theta=180^{\circ}$, 3) $\theta=90^{\circ}$; $-\ldots-$ спектр поглощения: 4) при комнатной температуре, 5) при $\kappa T=0,125 R$. Спектры нормированы на $2 \sqrt{\pi} h \Gamma$ $\left(\omega_{D}\right.$ - дебаевская частота, $\hbar \Gamma$ - полуширина спектра поглощения, $T$ - температура образца).

$\gamma$-кванты облучают вторую (не обязательно кристаллическую) мишень, работающую как резонансный поглотитель. Испущенные второй мишенью резонансные $\boldsymbol{\gamma}$-кванты регистрируются дискриминационным счетчиком. Исследуется скорость счета $I(\Omega)$ как функция ориентации $(\Omega)$ первой мишени по отношению к СИ.

Такая схема эксперимента позволяет регистрировать $\boldsymbol{\gamma}$-кванты, испускаемые возбужденными ядрами первой мишени в тот момент, когда они имеют максимальную по величине скорость в направлении второй мишени. Именно такие $\gamma$-кванты из-за эффекта Допплера обладают в лабораторной системе наибольшей энергией и с максимальной вероятностью поглощаются ядрами второй мишени. В геометрии рассеяния назад возбужденные ядра в первой мишени приобретают такую скорость через половину периода колебаний после поглощения кванта СИ. Из-за релаксации величина этой скорости $v_{1}$ всегда меньше начальной скорости $v_{0}=E_{0} / M c$ (в дебаевской модели $v_{1} \approx 0,75 v_{0}$ ). Потеря скорости $\Delta v=v_{0}-v_{1}$ зависит от направления $\mathbf{v}_{0}$ по отношению к соседним ядрам. Такая зависимость имеет место в кристаллах как низкой, так и высокой симметрии. В частности, в кубических кристаллах она возникает из-за эффектов ангармонизма, существенных при $R \geqslant 1$ эB. Чем больше $\Delta v$, тем меньше перекрытие спектра ГЯФ со спектром поглощения. Поэтому скорость счета фотонов дискриминационным детектором должна зависеть от $\Omega$. Оценка показывает, что с изменением $\Omega I(\Omega)$ может измениться в десятки и сотни раз (после исключения фона). Из сказанного следует, что функция $I(\Omega)$ содержит информацию о движении ядер в кристалле и прежде всего о релаксацин их кинетической энергии как функции направления движения. 
Укажем на некоторые особенности предложенной схемы, облегчающие получение эффекта. Использование $\gamma$-резонансов с энергией $0,2-1$ Мэв и временем жизни $10^{-11}-10^{-14}$ сек обеспечивает высокое сечение поглощения $\gamma$-квантов в фононном крыле $\sigma_{\Phi} \sim 10^{-21}-10^{-24} \mathrm{~cm}^{2}$, существенно превышающее сечение рассеяния электронной оболочкой. Кроме того, геометрия рассеяния назад на первой, а если необходимо, и на второй мишени с использованием дискриминационного детектора фотонов позволяет исключить паразитный фон комптоновского рассеяния *. Фон рэлеевского рассеяния (от двух мишеней) должен быть мал, поскольку его сечение на 5-6 порядков меньше $\sigma_{\phi}$. Поэтому эксперимент по данной схеме в принципе может проводиться без предварительной монохроматизации СИ. Использование подогрева второй мишени до $T \sim 1000^{\circ} \mathrm{C}$ может позволить проводить эксперименты на резонансах с энергией до нескольких Мэв.

Оценим скорость счета полезного сигнала. Примем в качестве интенсивности СИ в области $E \sim 0,3$ Мэв $10^{8}-10^{10} \kappa \boldsymbol{\kappa} /$ сек на мишень размером $10 \times 10$ м. $^{2}$ в энергетическом интервале $\sim 0,1$ эB. Принимая для двукратного рассеяния назад геометрический фактор ослабления $10^{-6}$ и учитывая, что лишь около $1 \%$ спектра ГЯФ перекрывается со спектром поглощения, получаем скорость счета $10-1000$ кв/сек.

В заключение отметим, что по предложенной схеме можно также исследовать ГЯФ низкоэнергетических $\gamma$-уровней $\left(E_{0} \leqslant 0,2\right.$ Мэв) с малым временем жизни $\left(\tau_{0} \leqslant 10^{-10}\right.$ сек). В случае таких $E_{0}$ спектр ГЯФ может заметно перекрываться со спектром поглощения и при расгеянии на углы, отличные от $\pi$ или нулевого угла. Это позволяет исследовать ГЯФ по зависимости сигнала от $\theta$ (использование монокристаллической мишени не обязательно). Имеются, однако, два усложняющих фактора. Во-первых, в случае $E_{0} \leqslant 0,2$ Мэв обычно $\tau_{0} \sim 10^{-10}$ сек $\gg \tau_{\text {эн }}$. Поэтому доля ГЯ $Ф$ в спектре рассеяния мала, а паразитный фон от обычной флюоресценции относительно велик. Тем не менее использование низких температур (следует охлаждать обе мишени) может существенно подавить такой фон, поскольку фононное крыло спектра обычной флюоресценции при $T=0 \mathrm{~K}$ не перекрывается с фононным крылом спектра поглощения, а бесфононное (мессбауэровское) излучение в данной схеме эксперимента заметного вклада не дает из-за его реабсорбции в первой мишени. Во-вторых, следует иметь в виду, что с уменьшением $E_{0}$ и $\theta$ труднее становится исключить фон комптоновского рассеяния. Поэтому в области $E_{0} \leqslant 0,2$ Мэв требуется предварительная монохроматизация СИ.

* При однократном комптоновском рассеянии назад энергия вторичных квантов не превышает 0,256 Мэв, а при двукратном - 0,128 Мэв.

\section{Л ИТЕРАТ У РА}

1. Кулипанов Г. Н., Скринский А. Н., Успехи физ. наук, 122, вып. 3, 369418 (1977).

2. Artemiev, A. N., Kabannik, V. A., Kazakov, Y., Kulipanov, G. N., Meleshko, E. A., Sklyarevskii, V. V., Skrinsky, A. N., Stepanov, E. P., Klestov, V. B., Chechin, A. J., J. de Physique, colloque C2, Suppl., 40, № 3, 23 (1979).

3. Х а а с М. А., Х и жняко в В. В., Ж. эксперим. и теор. физ., 74, вып. 1, 333-343 (1978).

Ннститут физики

Академии наук Эстонской ССР
Поступила в редакцию 15/IV 1980 